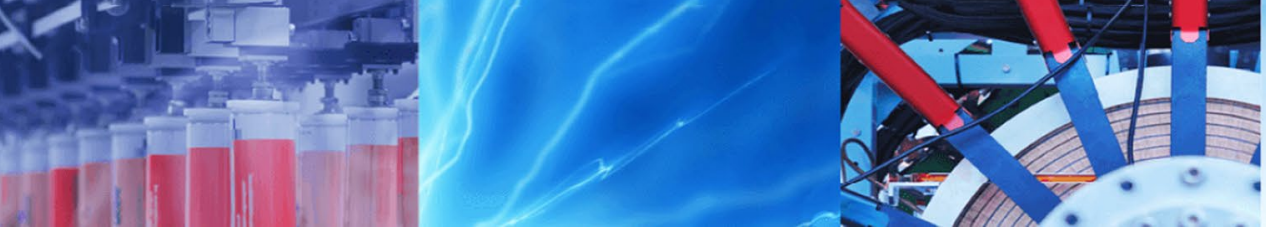

Research Article

\title{
Mechanical behavior of TPMS-based scaffolds: a comparison between minimal surfaces and their lattice structures
}

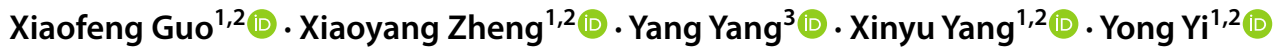

(c) Springer Nature Switzerland AG 2019

\begin{abstract}
Triply periodic minimal surfaces (TPMS) have drawn widespread attention out of their biomimetic features. This work compares skeletal-TPMS lattices with of strut-TPMS lattices in terms of their geometric and mechanical properties. Skeletal-TPMS lattices consist of continually smooth surfaces, while their counterparts, strut-TPMS lattices, are composed of cylindrical beams. These lattices derived from four kinds of TPMS, namely Gyroid (G), Schwarz Diamond (D), Schwarz Primitive $(\mathrm{P})$, and $\mathrm{iWp}(\mathrm{W})$, having incremental nodal connectivity of $3,4,6$, and 8 , respectively. The scaling laws of Young's modulus and yield strength are determined as power functions of volume fraction according to finite volume method (FVM) simulations. Their uniaxial compression behaviors are characterized by FVM simulations and are experimentally validated using additive manufacturing and uniaxial compression tests, showing that skeletal-TPMS lattices exhibit more homogeneous stress distribution. This work would not only broaden the material space for scaffolds, but shed light on the design criteria for tissue engineering.
\end{abstract}

Keywords Porous materials · Minimal surfaces · Architected materials · Finite volume method · Additive manufacturing

\section{Introduction}

Tissue engineering is an emerging branch of biomedical engineering. The purpose of tissue engineering research is to design and fabricate specifically-shaped degradable materials in which tissue cells are planted, corresponding tissue forms after a certain period, and is then implanted into the body [1]. As these materials gradually degrade in the body, the implanted tissue survives and begins to function. In the experiments of in vitro culture, three-dimensional cell growth is necessary, and a stable chemical micro-environment must be maintained through a specially-designed mass transfer process [2]. Such a design uses a biodegradable grid or a porous medium whose surface is specially treated as the growth framework. Thus, the design and preparation of three-dimensional framework is the foundation of tissue engineering $[3,4]$. One of the critical issues in orthopaedic regenerative medicine is the design of bone scaffolds and orthopaedic implants that replicate the biomechanical properties of the host bone [5]. Due to their adjustable stiffness and porosity, porous scaffolds are suitable for repairing or replacing damaged bone. Current orthopaedic implants are generally made of titanium and chromium alloys or stainless steel which are relatively stiff when compared to bones. This stiffness mismatch can generate stress shielding between the implant and surrounding bones. It also leads to premature implant loosening and implantation failure. Porous scaffolds can maintain cells survival and induce bone regeneration, with good biocompatibility $[6,7]$. Furthermore, in order to better control the degradation rate of materials and the potential of inducing

$\triangle$ Yong Yi, yiyong@swust.edu.cn; Xiaofeng Guo, guoxiaofeng14921@163.com; Xiaoyang Zheng, xyzheng1995@gmail.com; Yang Yang, 18683664536@163.com; Xinyu Yang, yangxinyu1128@163.com | 'State Key Laboratory for Environment-friendly Energy Material, Southwest University of Science and Technology, Mianyang 621000, China. ${ }^{2}$ Material Science and Engineering School, Southwest University of Science and Technology, Mianyang 621000, China. ${ }^{3}$ School of Economics and Management, Southwest University of Science and Technology, Mianyang 621000, China. 
bone marrow mesenchymal stem cells to differentiate into osteoblasts and promote their proliferation, porous materials need good bone conduction and bone inducement, respectively. However, an ideal porous scaffold requires pores in suitable size and porosity akin to human bones. At the same time, connected pores not only facilitate the adhesion and growth of the cells but also promote the growth of new bones, the transportation of nutrients, and the excretion of metabolites. In addition, satisfactory mechanical properties, including elastic modulus, Poisson's ratio, and yield strength, are required to ensure that the porous scaffolds can withstand long-standing loads under different environments.

Common preparation methods for porous materials, including phase inversion, phase separation, solventinduced pores, which are complex in operation and low in automation, cannot accurately control the size and distribution of pores. However, additive manufacturing (AM) shows great potential for tissue engineering applications with its ability to make predetermined external shape and internal architecture with very high level of accuracy and repeatability [8]. One of the most crucial superiorities is that porous materials printed by the AM not only have good biocompatibility but also meet the high requirements of mechanical properties and geometric structures. Moreover, the introduction of CAD technology has given the feasibility of the structural design and performance control of porous materials. By combining with metal AM, porous stents for orthopaedic implants not only enable the design and manufacture of individual implants, but also design porous structures within the prosthesis so that cells in the patient's bone can grow easily in the prosthesis and promote healing. All in all, AM has made a significant contribution to the design and fabrication of porous scaffolds [9].

Triply periodic minimal surfaces (TPMS), a kind of threedimensional continuously smooth surfaces, are mathematically known to minimize localized regions [10-12]. It divides the space into two intertwined complex regions whose sum of curvatures at each point on the surface is zero (i.e. the average curvature is zero). Its area is minimal under certain boundary conditions. And there is a tendency for TPMS to maintain its lowest energy in nature; thus such a surface often occurs since an increase in area requires additional surface energy. These structures are mainly characterized by the following characteristics: (1) Biomimetic structures: These structures are often found in biological systems in nature, such as the weevils, butterfly wings and shells of the beetles, and are therefore often used as biomimetic structures for biomedical purposes; (2) Long-range order: These structures are characterized by cubic symmetry and maintain the advantages of light weight, high strength and high specific surface area; (3) Mathematical definition:TPMS can be generated by mathematical modeling using implicit equations. When biomaterials are designed and fabricated with TPMS, their properties will be greatly enhanced. With this in mind, we have used TPMS solids as scaffold structures and found that they have great potential for tissue engineering applications. TPMS-based topologies have proven to be a more versatile source than the currently reported biomorphic scaffold designs [13, 14], providing a viable environment for the recovery and regeneration of damaged tissue cells because of the smooth bending properties of the TPMS surface and optimized fluid permeability [13]. Therefore, the geometric characteristics of TPMS have great prospects in the versatile design of structural systems. With the gradual development of AM technology, it has successfully provided a high-precision method for the manufacture of TPMS porous supports $[15,16]$.

In this paper, we design four sorts of skeletal lattices and strut lattices, respectively, taking inspiration from four kinds of TMPS (i.e. G, D, P, and W) [17]. These four lattices exhibit a three-dimensional bicontinuous interpenetrating ligament-channel structure and have interconnectivity orders of $3,4,6$, and 8 , respectively. To begin with, we analyze the geometric parameters (i.e. relative density, specific surface area, ligament diameter) of these lattices. The effective Young's modulus and yield strength based on relative density are obtained via FVM simulations. The simulation results are verified experimentally by $A M$ and uniaxial compression tests.

\section{Simulation method}

\subsection{Modeling and visualization}

Based on the trigonometric functions of these topologies, we used the solution of the scalar function of three independent variables to represent the value of TPMS. The three independent variables can be thought of as the $x, y$, and $z$ coordinates of the points in the three-dimensional Euclidean space. The model was constructed by changing the level parameter $t$ and the unit cell size. (The following are the definition equations for TPMS of G, D, P, and W) [18].

$$
\begin{aligned}
& \cos x \sin x+\cos y \sin z+\cos z \sin x=0 \\
& \sin x \sin y \sin z+\sin x \cos y \cos z \\
& \quad+\cos x \sin y \cos z+\cos x \cos y \cos z=0 \\
& \cos x+\cos y+\cos z=0 \\
& 2(\cos x \cos y+\cos y \cos z+\cos z \cos x) \\
& \quad-(\cos 2 x+\cos 2 y+\cos 2 z)=0
\end{aligned}
$$

\section{SN Applied Sciences}


The continuous minimal surfaces pinch off into unconnected blobs as the value of the parameter $t$ becomes sufficiently negative or positive. At such pinch points the smaller of the volume fractions remains considerable. Hence, the equations for the skeletal-TPMS structures that generate $G, D, P$, and $W$ are:

$\mathrm{F}(\mathrm{x}, \mathrm{y}, \mathrm{z})=\mathrm{a}_{1} \mathrm{~T}_{1}+\mathrm{a}_{2} \mathrm{~T}_{2}+\mathrm{t}$

To reduce the complexity, set $a_{1}$ and $a_{2}$ to constants, and $T_{1}$ and $T_{2}$ are one of the expressions for the very minimal surfaces of $G, D, P$, and $W$. The horizontal parameter $t$ is a variable that determines the volume fraction associated with the area separated from the surface. Therefore, the skeletal-TPMS functions of $G, D, P$, and $W$ are defined as [19]:

$$
\begin{aligned}
\mathrm{F}(\mathrm{G})= & 10(\cos x \sin x+\cos y \sin z+\cos z \sin x) \\
& -0.5(\cos 2 x \cos 2 y+\cos 2 y \cos 2 z+\cos 2 z \cos 2 x)+t
\end{aligned}
$$

$$
\begin{aligned}
\mathrm{F}(\mathrm{D})= & 10\left(\sin x-\frac{\Pi}{4} \sin y-\frac{\Pi}{4} \sin z-\frac{\Pi}{4}+\sin x\right. \\
& -\frac{\Pi}{4} \cos y-\frac{\Pi}{4} \cos z-\frac{\Pi}{4}+\cos x-\frac{\Pi}{4} \sin y \\
& \left.-\frac{\Pi}{4} \cos z-\frac{\Pi}{4}+\cos x-\frac{\Pi}{4} \cos y-\frac{\Pi}{4} \sin z-\frac{\Pi}{4}\right) \\
& -0.7(\cos 4 x+\cos 4 y+\cos 4 z)+t
\end{aligned}
$$

$\mathrm{F}(\mathrm{P})=10(\cos x+\cos y+\cos z)$

$$
\begin{aligned}
& -5.1(\cos x \cos y+\cos y \cos z \\
& +\cos z \cos x)+t
\end{aligned}
$$

$$
\begin{aligned}
\mathrm{F}(\mathrm{W})= & 10(\cos x \cos y+\cos y \cos z+\cos z \cos x) \\
& -5(\cos 2 x+\cos 2 y+\cos 2 z)+t
\end{aligned}
$$

The geometry of the Skeletal-TPMS consists of are ligaments. The routines are written in the language of MAT$L A B$, and the isosurfaces of these skeletal structures are modeled by the software MATLAB (Ver.2016b). Commands based on the skeletal structure function save the vertex and face list as an object (.OBJ) file format that can be opened and re-edited by other 3D graphics application vendors. Then we import the .OBJ file format into Autodesk 3ds Max software (Ver.2016) to cap the boundaries of the isosurface. Subsequently, skeletal structure was exported as a series of stereolithography (.STL) file formats for computational simulation and AM (See Fig. 1a).

The geometry of the strut-TPMS lattices consists of cylindrical beams. Instead of parametric modeling, CADbased software, SolidWorks, is used to generate strut-TPMS lattices. Firstly, the frameworks of lines of strut-TPMS lattices are generated according to their nodal connectivity and ligament orientation. Next, the frameworks are transformed into 3D solid skeletons via lofting based on a circle with different diameter that are used to determine their relative density. Finally, a single or multiple-cell lattice are created by combining 3D solid skeletons with a box in intersection Boolean operation. It is noteworthy that each strut-TPMS lattice can be tuned by the diameter of the circle used as its ligament diameter, as well we the
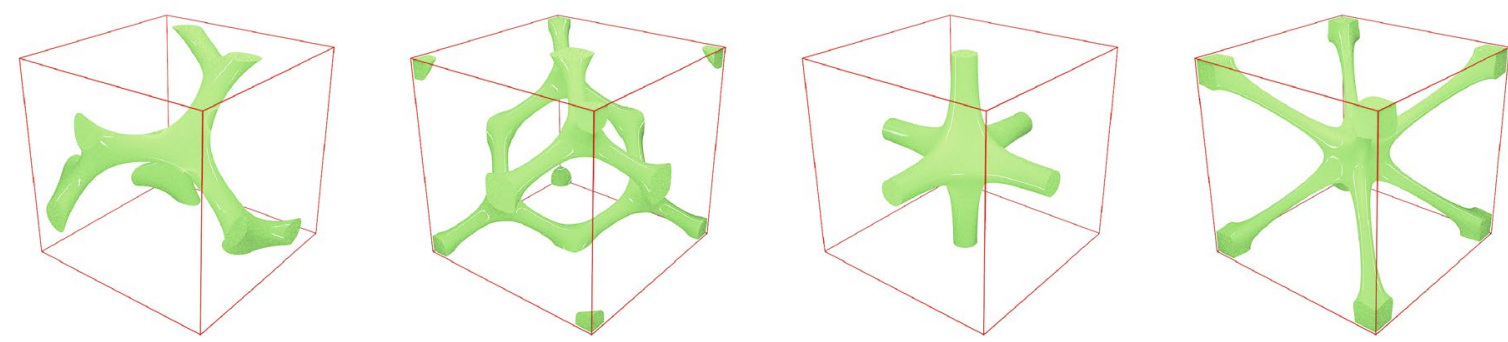

(b)
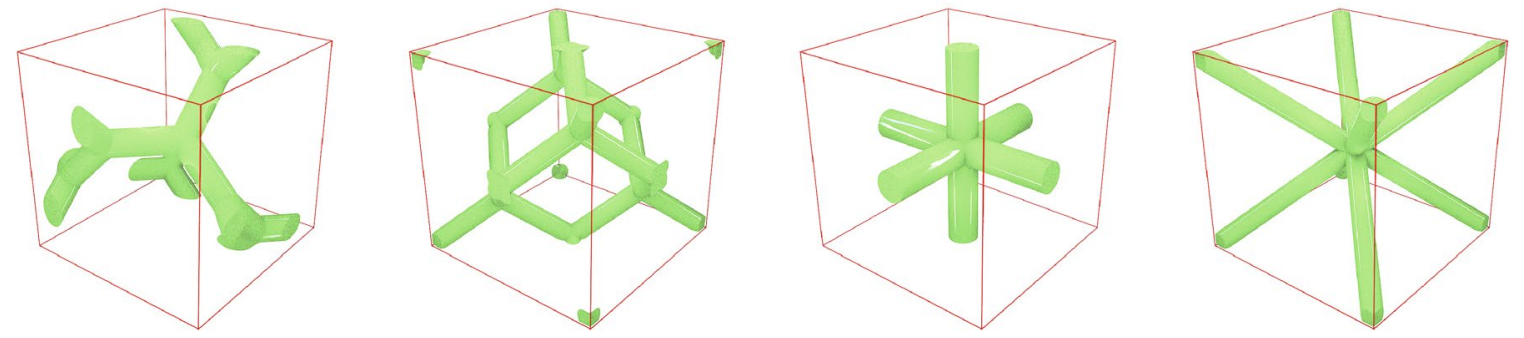

Fig. 1 Architectures of skeletal-TPMS (a) and strut-TPMS (b) 
length and cell number of skeletons. Similarly, strut structures are exported as a series of stereolithography (.STL) (see Fig. 1b).

\subsection{Finite volume method simulation}

We use a commercial package, GeoDict (Ver.2017) [20], to perform FVM simulations to probe the effective mechanical properties (i.e. Young's modulus and yield strength) of these topologies. Compared with finite element method, which is more common in terms of computer simulation, FVM is faster and more efficient with respect to the simulations of these complicated porous materials [21-28].

As for the mechanical performance simulations used in this paper, we used the ElastoDict-VOL and ElastoDictLD modules to calculate the effective stiffness (Young's modulus) and the effective strength (yield strength) [29]. The stiffness is derived from the calculation of the linear elastic deformation of porous structure, while the strength is a nonlinear large deformation. In fact, based on the theory of continuum mechanics, it was found that the size of the structure used in FVM calculation (i.e. the average ligament size) has no size effect or surface effect because of the unstressed sample boundary [30, 31]. This means that in our simulations, simply magnifying or reducing the size of the model does not change the calculations (i.e. Young's modulus and yield strength). In addition, all simulation calculations were based on under periodic boundary conditions. Maskery et al. developed a robust finite element model to determine the useful numerical relationship between their moduli and volume fractions. In particularly, their study of the lattice functional grading can accurately predict the elastic modulus of a graded density lattice [32]. Furthermore, Maskery et al., through experiments and finite element calculations, clarified the deformation mechanisms of the lattices based on TPMS printed by AM [33]. Compared to their calculation work, we used the unit cell as the smallest representative volume element. That is to say, the calculated elastic modulus is equal to the equivalent elastic modulus of the infinite unit cell structure.

Since the voxel resolution determines the result accuracy of FVM simulations, we firstly carried out a grid study by varying the numerical grid to estimate the uncertainty of FVM. To this end, we calculated the effective Young's modulus of skeletal-P lattices with relative density ranging from 0.05 to 0.2 . The relative error is defined by $\delta E=\left(E_{n}-E_{n-1}\right) / E_{n}$, where $E_{n}$ is the current value of Young's modulus and $E_{n-1}$ is the former one. Figure 2 shows that the domain size dominates the accuracy of FVM, demonstrating a relative error lower than $0.1 \%$ under refined domain size above $250 \times 250 \times 250$. Thus, in this work, a fixed domain size of $300 \times 300 \times 300$ is set for all the simulations in consideration of accuracy and computation time.

\section{Experimental method}

\subsection{Specimen preparation}

In order to compare the mechanical differences between skeletal-TPMS and strut-TPMS, we used an industrial scale 3D printer EOS $P 110$ to fabricate a set of topologies with an identical relative density of 0.1 (see Fig. 3). This innovative manufacturing platform is able to produce such

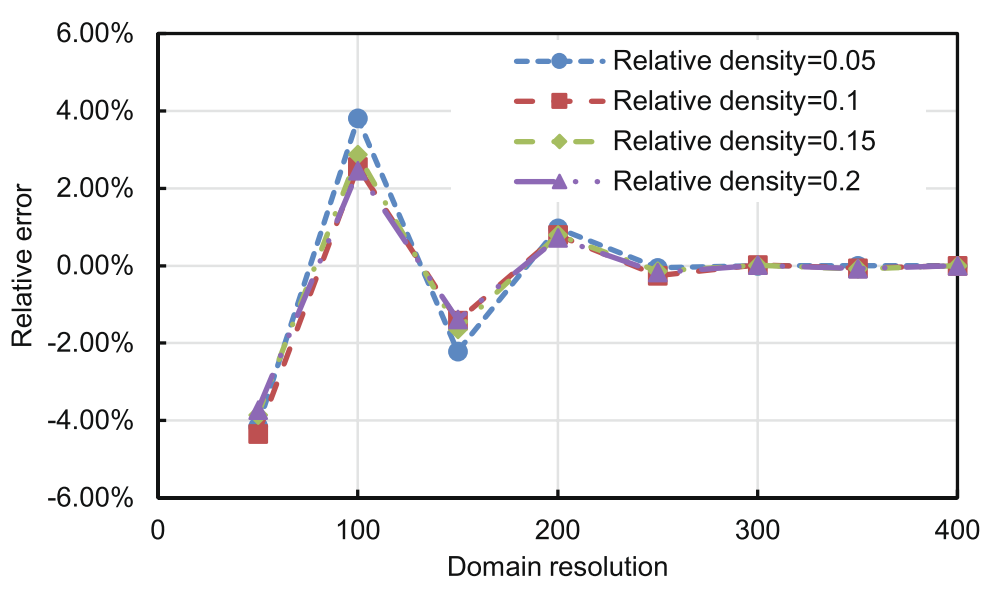

(a)

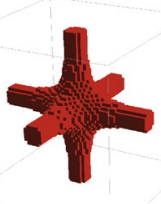

(b)

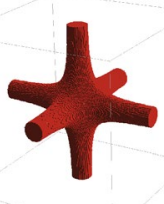

(d)

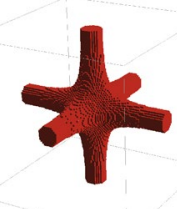

(c)

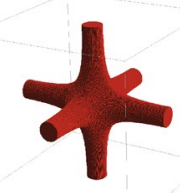

(e)

Fig. 2 The effect of domain resolution on simulation uncertainties (a); voxel images of Skeletal-P used to the indication of uncertainties, the domain size is $50 \times 50 \times 50$ (b), $100 \times 100 \times 100$ (c), $200 \times 200 \times 200$ (d), and $300 \times 300 \times 300$ (e), respectively 
complicated topologies without support, meanwhile keeping dimensional accuracy and reproducibility. Whitish fine powder PA 2200 based on polyamide 12 was used as raw materials by virtue of its excellent material properties including high strength, high stiffness, good chemical resistance, detail resolution and good biocompatibility. These models consist of $4 \times 4 \times 4$ unit cells with the dimension of $40 \mathrm{~mm} \times 40 \mathrm{~mm} \times 40 \mathrm{~mm}$ and layer thickness of $0.05 \mathrm{~mm}$. Such settings are not limited by generality and are sufficient to present the resolution of the structural features. The dimensions were chosen to reduce any possible effects of introducing loads along the z-axis in the elastic and elastic-plastic simulations and to adjust them within acceptable resolution.

\subsection{Compression testing}

We used a static testing machine (MTS Criterion TM Model 45, $30 \mathrm{kN}$ load cell) to perform a static compression test at a constant deformation rate of $1 \mathrm{~mm} / \mathrm{min}$ to obtain the mechanical properties of the 3D printed model. The compression test was carried out in accordance with ASTM standard D695-15 (plastic) [34]. We obtained the stress-strain curve for each printed topology, and then calculated the Young's modulus from the slope of the linear deformation in the elastic deformation. The yield strength was defined as the $1 \%$ offset stress based on the shape of the stress-strain curve.

\section{Results and discussion}

\subsection{Geometrical properties}

As mentioned earlier, we analyzed the geometric properties (relative density, specific surface area, and ligament

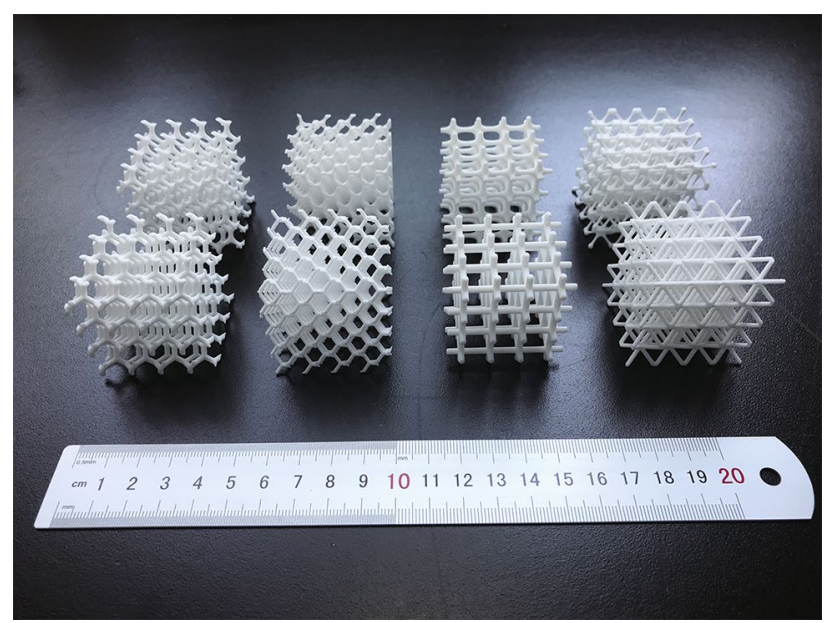

Fig. 3 3D-printed skeletal-TPMS (top) and strut-TPMS (bottom) diameter) of these lattices. The scaling laws of the four sorts of skeletal-TPMS lattices based on relative density are as follows:

$\rho_{\text {ske }}(\mathrm{G})=0.03315 \mathrm{t}+0.4972$

$\rho_{s k e} \mathrm{D}=0.03972 \mathrm{t}+0.4892$

$\rho_{\text {ske }}(\mathrm{P})=9.309 * 10^{-5} t^{3}+9.607 * 10^{-4} t^{2}+3.238 * 10^{-2} t+0.5925$

$\rho_{\text {ske }}(\mathrm{W})=6.729 * 10^{-4} t^{2}+2.333 * 10^{-2} t+0.5258$

Here, when the parameter $t$ is equal to zero, the initial values of the relative densities of the four structures are $\rho_{\text {ske }}(\mathrm{G})$ initial $=0.4972, \rho_{\text {ske }}(\mathrm{D})$ initial $=0.4892, \rho_{\text {ske }}(\mathrm{P})$ initial $=0.5925$, and $\rho_{\text {ske }}(\mathrm{W})$ initial $=0.5258$. Among them, the relative density of $G$ and $D$ structures is obviously linear, and $\mathrm{P}$ and $\mathrm{W}$ are polynomial function relations.

Since these four sorts of strut-TPMS lattices are not modeled by parametric equations, their scaling laws based on relative density are as follows:

$\rho_{\text {stru }}(G)=1.683 \times d^{* 2}+0.3554 d^{*}-0.02479$

$\rho_{\text {stru }}(\mathrm{D})=3.635 \times d^{* 2}+0.1684 d^{*}-0.00295$

$\rho_{\text {stru }}(\mathrm{P})=1.493 \times d^{* 2}+0.1729 d^{*}-0.0109$

$\rho_{\text {stru }}(\mathrm{W})=4.698 \times d^{* 2}-0.154 d^{*}+0.01823$

Similarly, when the relative densities are equal, the two important geometrical characteristics of the ligament diameter and the specific surface area are quite different.

It is well known that the ligament diameter is the basic structure connecting adjacent nodes, which closely affects the structural and geometric properties of porous scaffolds. It is a necessary reference for the printing process. Due to the limitation of AM resolution, in order to obtain higher precision, it is usually implemented by printing a version slightly larger than the desired object with standard resolution. Therefore, for more convenient determination of the ligament diameter, the diameter of the ligament is defined as $d^{*}$ :

$d^{*}=d_{\text {lig }} / /$

where $d_{\text {lig }}$ defined as the diameter value of the middle ligament of the two nodes of each single unit, and I defined as the side length of these topologies. The following are the scaling laws of ligament diameter for the four sorts of skeletal-TPMS lattices:

$d_{s k e}^{*}(G)=0.6693 \rho^{0.7072}+0.03198$

$d_{s k e}^{*}(D)=0.6440 \rho^{0.6905}+0.04195$

$d_{s k e}^{*}(P)=1.017 \rho^{0.5018}-0.02578$ 


$$
d_{s k e}^{*}(W)=0.5882 \rho^{0.7498}+0.09153
$$

The following are the scaling laws of ligament diameter for the four sorts of strut-TPMS lattices:

$d_{\text {stru }}^{*}(G)=0.6890 \rho^{0.6325}+0.02859$

$d_{\text {stru }}^{*}(D)=0.5169 \rho^{0.5336}-0.00468$

$d_{\text {stru }}^{*}(P)=0.7807 \rho^{0.5722}+0.01166$

$d_{\text {stru }}^{*}(W)=0.5097 \rho^{0.3863}-0.06006$

Figure 4a shows that the ligament diameters of the eight topologies are proportional to the relative density. At a fixed relative density, the ligament diameter of the strut-TPMS lattices is thicker than that of the skeletal-TPMS lattices. In particular, the P structure has the highest ligament diameter among the four TPMS (when $\left.\rho=0.2, d_{\text {stru }}^{*}(P) \approx 0.3222, d_{\text {ske }}^{*}(P) \approx 0.2637\right)$.

The specific surface area refers to the total surface area of each scaffold. The larger the total surface area is, the more ingrowth the bone tissue cells will have, which makes the cells to be cultured more easily in the porous structure [35]. Since these topologies isosurfaces subject to translation and scaling, here we use a particular dimensionless parameter $s^{*}$ to represent the specific surface area:

$s^{*}=S I / V$

Here, the outer surfaces are neglected due to the threedimensional periodic characteristics. $S$ represents the inner surface area of the isosurface. In addition, $I$ and $V$ are the side length and the skeletal volume of these topologies, respectively. When the side length / is a certain value, a lower relative density could result in a lower specific surface area.
In addition, by changing $I$, these skeletal isosurfaces can be translating and scaling to nanoscale, and excellent high specific surface area could also be obtained. Besides, we can change the length / of the same topological structure, the same special surface area value can be obtained by the formula $S I N$. If use the formula $S N$, by changing the length I, we will obtain different specific surface area values. The following are the scaling laws for the specific surface area of the four sorts of skeletal-TPMS lattices:

$s_{s k e}^{*}(G)=-27.12 \rho^{2}+14.04 \rho+0.8574$

$s_{s k e}^{*}(D)=-21.75 \rho^{2}+16.43 \rho+1.134$

$s_{s k e}^{*}(P)=-17.50 \rho^{2}+10.04 \rho+0.6287$

$s_{s k e}^{*}(W)=-24.64 \rho^{2}+14.42 \rho+1.008$

The following are the scaling laws for the specific surface area of the four sorts of strut-TPMS lattices:

$s_{\text {stru }}^{*}(G)=-25.34 \rho^{2}+13.7 \rho+0.8779$

$s_{\text {stru }}^{*}(D)=-28.79 \rho^{2}+16.81 \rho+1.144$

$s_{\text {stru }}^{*}(P)=-21.64 \rho^{2}+11.54 \rho+0.7185$

$s_{\text {stru }}^{*}(W)=-41.09 \rho^{2}+19.64 \rho+1.015$

Figure $4 \mathrm{~b}$ shows the relations between the specific surface area and relative density of these eight topologies. The specific surface area is proportional to the relative density, exhibiting a polynomial function. It can be clearly seen that the $D$ topology has a highest specific surface area among the four TPMS when the relative densities are identical (when $\rho=0.2, s_{\text {stru }}^{*}(D) \approx 3.359, s_{\text {ske }}^{*}(D) \approx 3.313$ ). Similarly, each strut-TPMS lattice has a higher specific

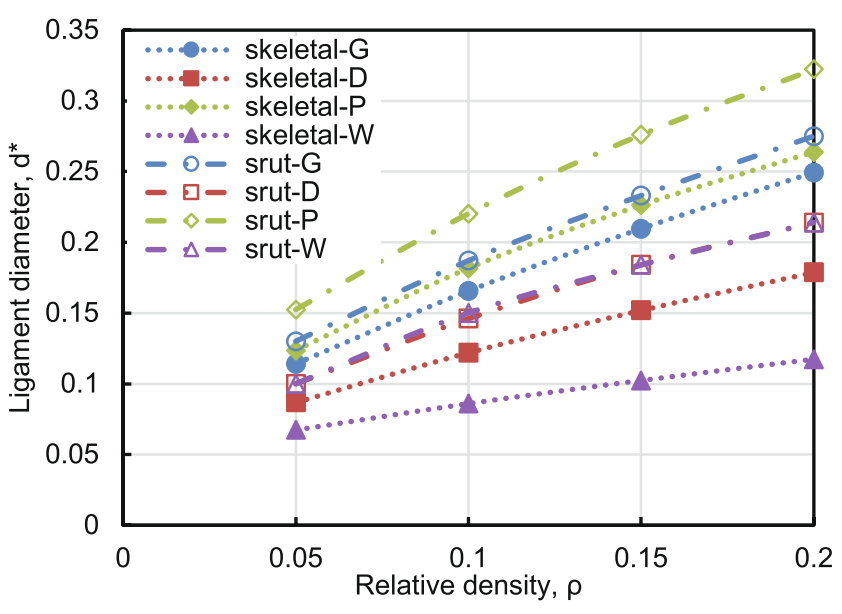

(a)

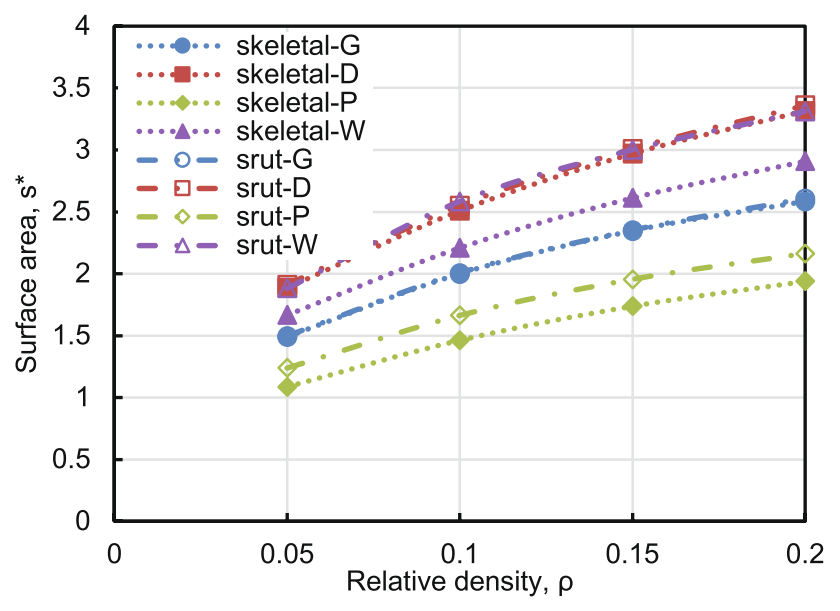

(b)

Fig. 4 The differences in ligament diameter (a) and in specific surface area (b) between skeletal and strut-TPMS 
surface area than its counterparts, the skeletal-TPMS lattice. In addition, strut-D and strut-W have a similar trend.

\subsection{Mechanical properties}

The mechanical properties of porous structures are highly valued when they are used as bone supports and orthopaedic implants, so it is necessary to explore the mechanical properties of these topologies. We mainly compare the Young's modulus and yield strength. In addition, since these topologies have no scale effect at the boundary, we use the classical scaling law of Gibson-Ashby for the opencell micro-foams to express the ratio of Young's modulus $E$ (Fig. 5a) and yield strength $\sigma$ (Fig. 5b) law [36]:

$$
\begin{aligned}
& E^{*} / E_{\text {lig }}=\rho^{2} \\
& \sigma^{*} / \sigma_{\text {lig }}=0.3 \rho^{1.5}
\end{aligned}
$$

Here, $E^{*}$ and $\sigma^{*}$ represent the Young's modulus and yield strength of the overall foam, respectively; $E_{\text {lig }}$ and $\sigma_{\text {lig }}$ are the Young's modulus and yield strength of the ligament material, respectively, and $\rho$ is the relative density. It is apparent that the relative Young's modulus $\left(E^{*} / E_{\text {lig }}\right)$ and the relative yield strength $\left(\sigma^{*} / \sigma_{\text {lig }}\right)$ vary with the relative density $\rho$, respectively. The following are the scaling laws of the Young's modulus and yield strength of the four sorts of skeletal-TPMS lattices:

$$
E_{\text {ske }}^{*}(G) / E_{\text {lig }}=0.9515 \rho^{2.174}
$$

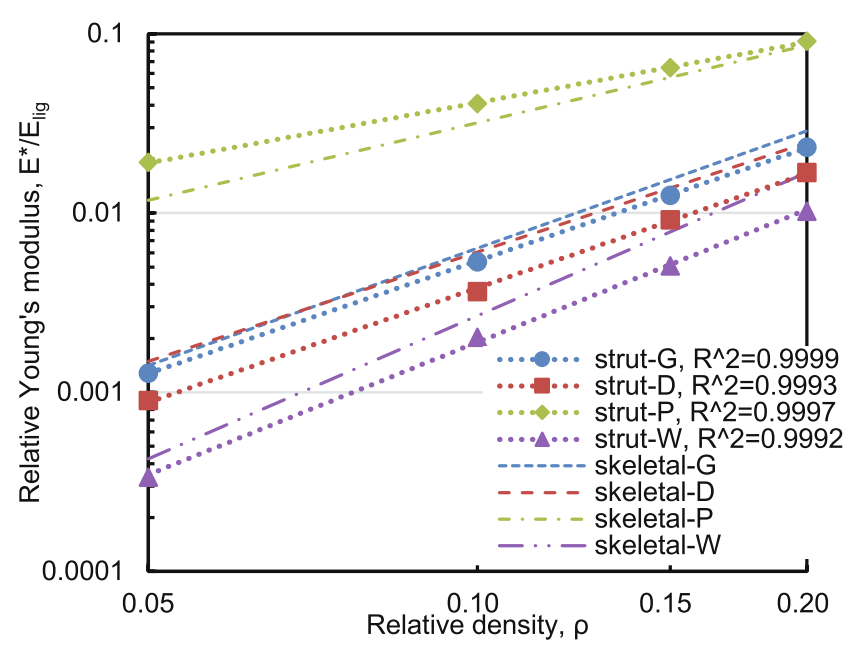

(a)

$$
\begin{aligned}
& E_{\text {ske }}^{*}(D) / E_{\text {lig }}=0.6438 \rho^{2.026} \\
& E_{s k e}^{*}(P) / E_{\text {lig }}=0.8707 \rho^{1.437} \\
& E_{s k e}^{*}(W) / E_{\text {lig }}=1.204 \rho^{2.654} \\
& \sigma_{\text {ske }}^{*}(G) / \sigma_{\text {lig }}=0.6182 \rho^{1.746} \\
& \sigma_{\text {ske }}^{*}(D) / \sigma_{\text {lig }}=0.6802 \rho^{1.614}
\end{aligned}
$$

$\sigma_{\text {ske }}^{*}(P) / \sigma_{\text {lig }}=0.4505 \rho^{1.250}$

$\sigma_{\text {ske }}^{*}(W) / \sigma_{\text {lig }}=0.8791 \rho^{2.124}$

The following are the scaling laws for the Young's modulus and yield strength of the four sorts of strut-TPMS lattices:

$E_{\text {stru }}^{*}(G) / E_{\text {lig }}=0.6657 \rho^{2.090}$

$E_{\text {stru }}^{*}(D) / E_{\text {lig }}=0.5050 \rho^{2.121}$

$E_{\text {stru }}^{*}(P) / E_{\text {lig }}=0.5439 \rho^{1.120}$

$E_{\text {stru }}^{*}(W) / E_{\text {lig }}=0.5437 \rho^{2.456}$

$\sigma_{\text {stru }}^{*}(G) / \sigma_{\text {lig }}=0.6177 \rho^{1.768}$

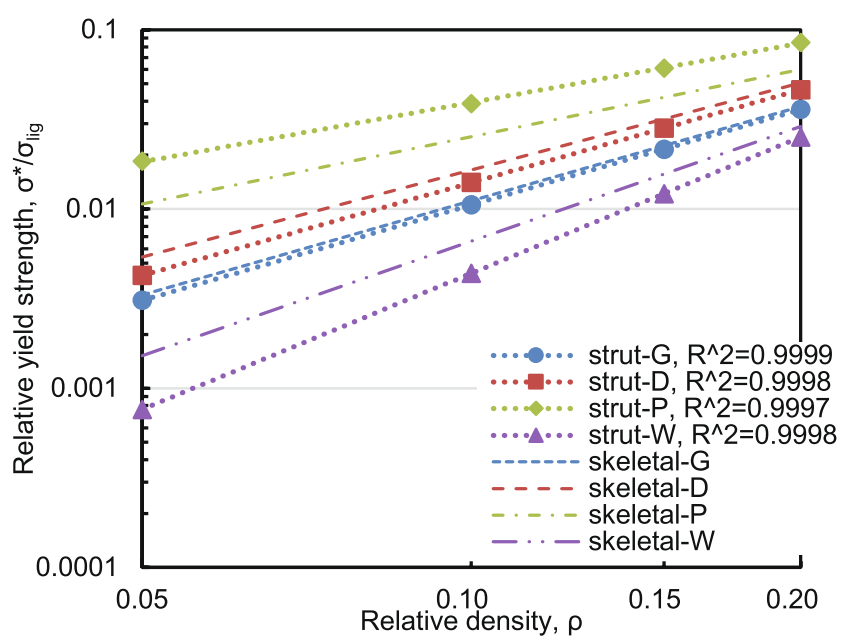

(b)

Fig. 5 The scaling laws of young's modulus (a) and yield strength (b) for skeletal and strut-TPMS 
$\sigma_{\text {stru }}^{*}(D) / \sigma_{\text {lig }}=0.7387 \rho^{1.721}$

$\sigma_{\text {stru }}^{*}(P) / \sigma_{\text {lig }}=0.4946 \rho^{1.100}$

$\sigma_{\text {stru }}^{*}(W) / \sigma_{\text {lig }}=1.4629 \rho^{2.524}$

The Young's modulus and yield strength of the ligament materials were calculated according to the simulation to obtain $E_{\text {lig }}=1.7 \mathrm{GPa}$ and $\sigma_{\text {lig }}=50 \mathrm{MPa}$. Moreover, it can be seen from the simulation and experimental graphics that when the relative density is less than 0.5 , the Young's modulus and yield strength of the P structure is the highest among these structures, indicating that it has the highest stiffest and strength. Meanwhile, $W$ structure have the lowest stiffness and strength although it has the largest connectivity of the 8 , which means that the larger number of nodes does not lead to higher rigidness.

The literature mention that the power law index $n$ is commonly used to evaluate the deformation behavior of porous materials $[37,38]$. The power law relationship has a great influence on the scaling law of porous structures. However, through simulation and experiment, it is concluded that the P structure is the least affected by the relative density, because the power law index $n$ in the Young's modulus $E^{*}$ of skeletal-P and strut-P is 1.437 and 1.120 , respectively, while other structures are over 2 . The power law index $n$ in the yield strength $\sigma^{*}$ for skeletal$P$ and strut- $P$ is 1.250 and 1.100 , respectively, while the power law index $n$ of other structures is greater than 1.6, indicating that $P$ structure is much strength than other structures. This is because the $P$ structure is the tensiledominant mode of deformation, and the other three structures are the bending-dominant mode of deformation. Furthermore, the W structure has the lowest elastoplastic properties. Among the four kinds of TPMS, the power law index $n$ in the Young's modulus $E^{*}$ of skeletal-W and strut$W$ is 2.654 and 2.456, respectively. The power law index $n$ in the yield strength $\sigma^{*}$ for skeletal-W and strut-W is 2.124 and 2.524, respectively. Therefore, the power law index $n$ of the $\mathrm{W}$ structure is higher than the other three structures. The data proves that the power law index has a significant impact on mechanical properties. The structural differences between the four sorts of skeletal-TPMS lattices and the four sorts of strut-TPMS lattices result in significant differences in their mechanical properties.

We applied a compressive deformation of 0.01 along the $z$-axis to the eight topologies. The von Mises stress map was obtained by von Mises staining (Fig. 6).
The eight $40 \mathrm{~mm} \times 40 \mathrm{~mm} \times 40 \mathrm{~mm}$ models with a unit cell number of $4 \times 4 \times 4$ have a relative density of 0.1 . However, under the compression, the stress that they need to deform from elastic state to plastic state is completely different, so is the deformation mechanism. Not all ligaments exhibit the same stress concentration, but exhibit distinctly different forms of principal stress distribution under the identical stress. In Fig. 6, skeletal-G presents a continuous form of stress concentration, which appears as a spiral strip, while strut- $G$ is a discrete point unit at the ligament junction. The stress concentration of skeletal-D is expressed as the ring of the middle part of the ligament, while the stress concentration of strut- $D$ is the discrete point unit at the node. The stress concentration of skeletal-P is present in the middle of the ligament, while the strut- $P$ is whole stressed at the ligament in the direction of the force. This also explains that strut-P has slightly higher mechanical properties than skeletal-P. Skeletal-W and strut-W also exhibit stress concentration at the joint position of the ligament, the difference being that the former is a smooth surface and the latter is a discrete point. In general, the yield points of the three structures $G, D$, and $W$ are quite different from those of the P structure. There is no prominent stress concentration, showing good elastoplastic, which is in good agreement with the simulation results. In addition, under the identical type of TPMS conditions, skeletal-TPMS generally exhibits more significant stress concentration than strut-TPMS except $P$ structure. Moreover, by comparing the Young's modulus and yield strength of skeletal-TPMS and strut-TPMS, it is found that the three skeletal-TPMS of G, D and W structures have higher stiffness and strength than strut-TPMS. The P structure is completely opposite, and its strut-TPMS has slightly higher mechanical properties than skeletalTPMS. In summary, it can be concluded that skeletal-TPMS has a more uniform and reasonable stress distribution than strut-TPMS.

\subsection{Compression behavior and failure mechanism}

In order to probe how the ligaments collapse under large deformation, uniaxial compression tests are performed and the resulting stress-strain curves of the eight 3D-printed topologies are shown in Fig. 7. The results show that the compression behavior consists of three stages. Firstly, the ligaments of all structures exhibit linear elastic compression behavior and good elastic deformation when they are subject to compression. Secondly, with further compression, the linear relationship between stress and strain is destroyed when the unidirectional stress is large enough to the yield point of materials. That is, the models 


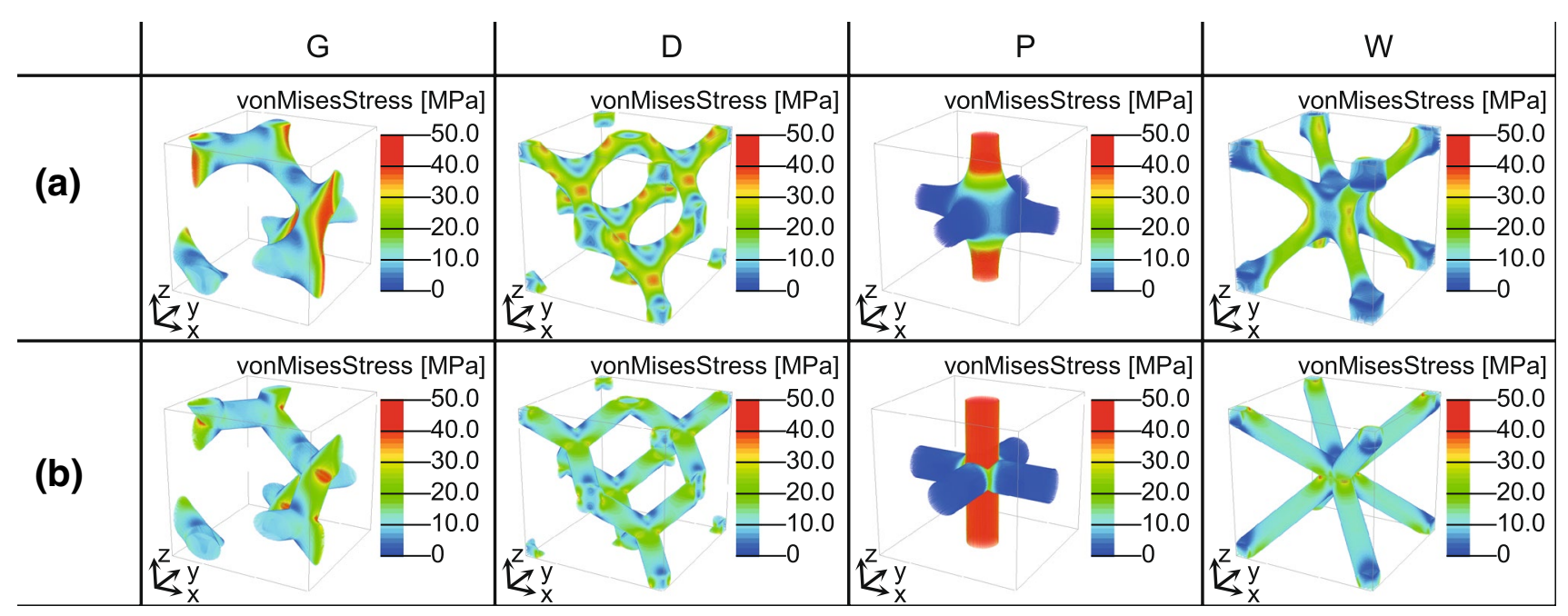

Fig. 6 Structural views of skeletal (a) and strut-TPMS (b) under 0.01 compressive deformation along z-axis (the color map indicates the von Mises stress)

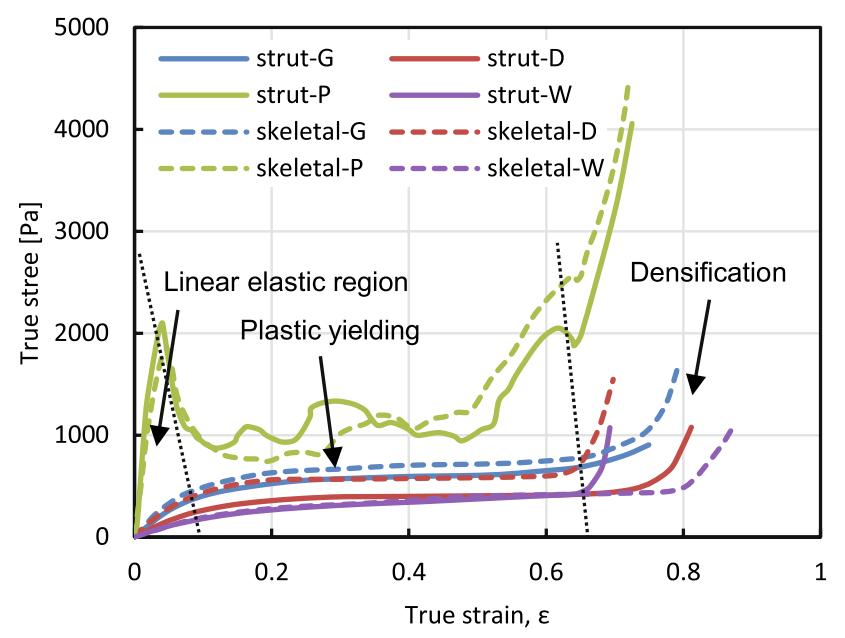

Fig. 7 Stress-strain curve from uniaxial compression mechanical testing

begin to enter the plastic state from the elastic state, and the plastic yield due to the collapse of the pores. The stress-strain curve presents a limited platform. Finally, in the third stage, the ligaments of the models begin to densify because the compressive stress exceeds the strength limit of the sample. That is, the failure effect occurs.

It can be seen from the uniaxial compression tests (see Figs. 8,9 ) and the stress-strain curve that the stress-strain response of the four basic topologies are very distinct. However, the P structure exhibits a disparate deformation behavior compared to the other three structures. The growth rate of skeletal-TPMS and strut-TPMS is very rapid at the linear elastic stage, and stress peaks appears. The stress is then rapidly reduced due to ligament bending and plastic deformation. The platform that causes the $P$ structure to fluctuate during the plastic deformation phase is different from the smoothing platform of the other three structures. This is because the direction of several ligaments in the $P$ structure are consistent with the direction of compression, and the structure express a tensile-dominant deformation behavior. The direction of ligaments in other three types of structures $G$, $D$ and $W$ have a certain angle with the compression direction, which makes them appear curved-dominant deformation behaviors. Moreover, it can be seen from the comparison between Figs. 8 and 9 that when the uniaxial (z-axis) stress is applied in the experiment, skeletal-P and strut-P exhibit different deformation behaviors. That is, the tensile-dominant deformation mode is that under uniaxial compression, the ligaments tend to be bent and fractured under stress. The other three types of structures have a larger plastic deformation path, that is, they can carry a greater degree of plastic deformation, and their pores are gradually closed from a spherical shape to an elliptical line under uniaxial compression.

\section{Conclusions}

In conclusion, we compare skeletal-TPMS lattices with strut-TPMS lattices with respect to their geometric (relative density, specific surface area and ligament diameter) and mechanical properties (Young's modulus and yield strength). According to FVM simulations and 

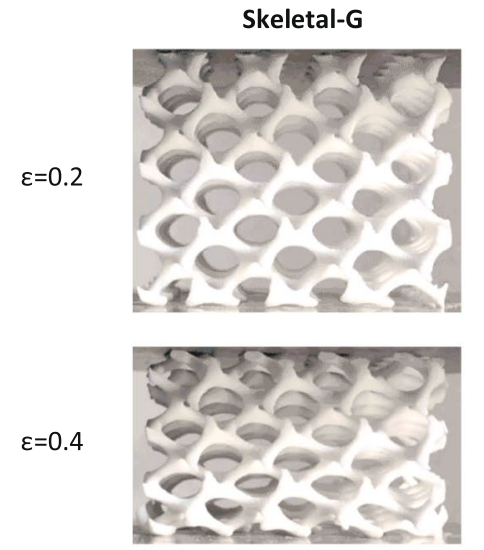

$\varepsilon=0.6$

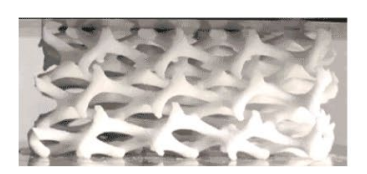

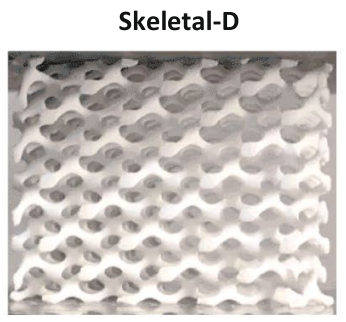
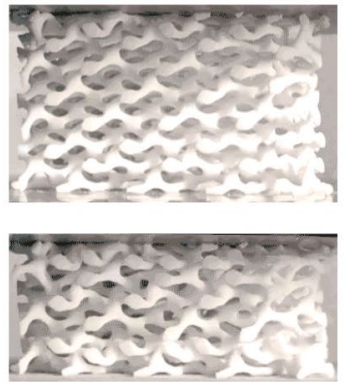
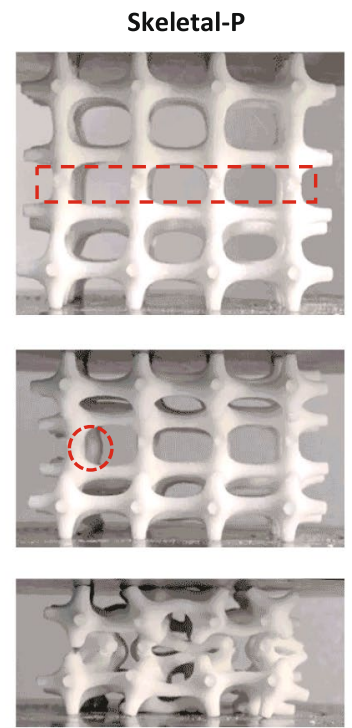
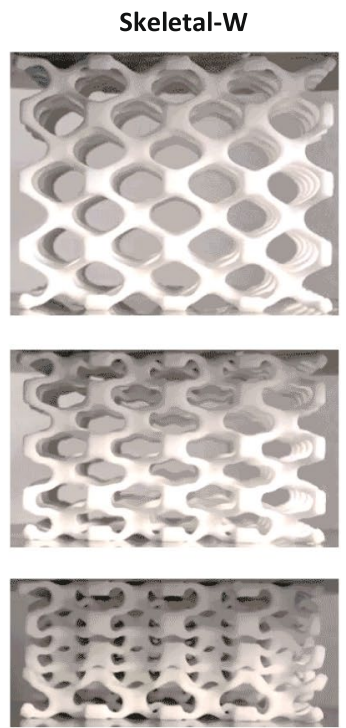

Fig. 8 Deformation of the 3D printed skeletal-TPMS model in uniaxial compression tests

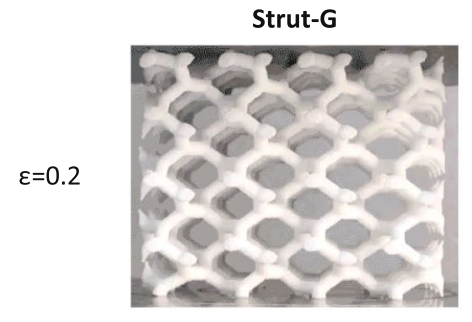

$\varepsilon=0.4$

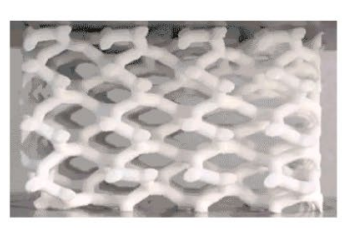

$\varepsilon=0.6$

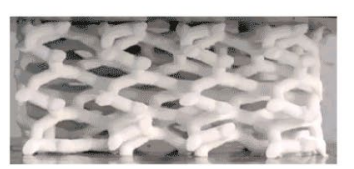

Strut-D
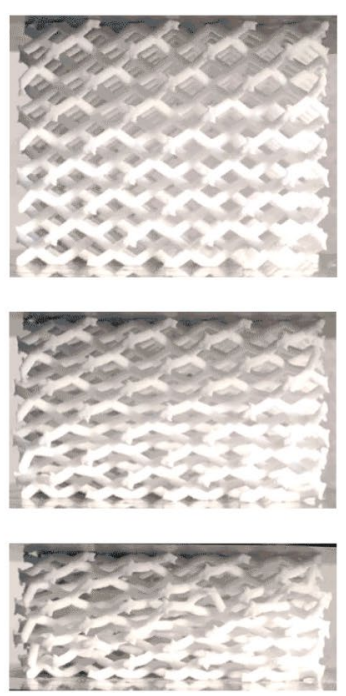

Strut-P
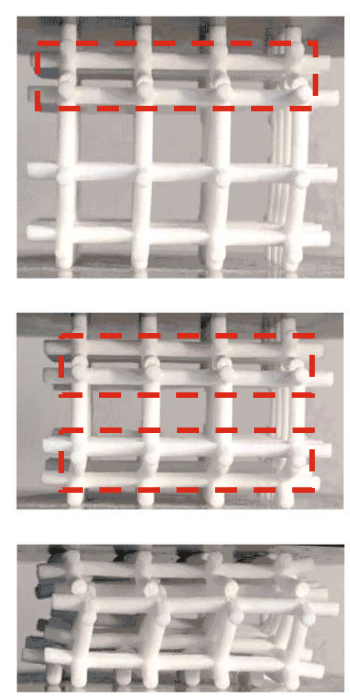
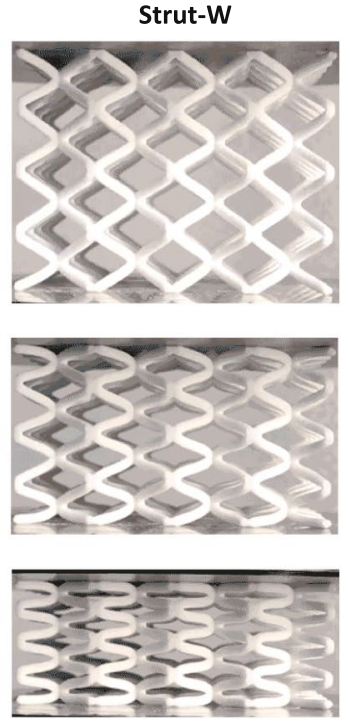

Fig. 9 Deformation of the 3D printed strut-TPMS model in uniaxial compression tests

experimentally uniaxial compression tests, the Young's modulus and yield strength of both of skeletal-TPMS and strut-TPMS lattices are calculated as functions of relative density, demonstrating that skeletal-TPMS and strutTPMS based on the same topology have a similar scaling law. Nevertheless, skeletal-TPMS lattices exhibit a more uniform, smooth-transition stress distribution than strutTPMS lattices, making them the promising candidates to tissue engineering. More importantly, this work provides a feasibility method for topology optimization design for scaffold design, with a view to achieving applications in bone engineering and orthopaedic implants.

Acknowledgements This work was supported by the Undergraduate Innovation Fund Project for Accurately Funded Special Projects of Southwest University of Science and Technology (SWUST) (jz19-001). 


\section{Compliance with ethical standards}

Conflict of interest The authors declare that they have no conflict of interest.

\section{References}

1. Chen G, Ushida T, Tateishi T et al (2002) Scaffold design for tissue engineering. Macromol Biosci 2(2):67-77

2. Mehdizadeh H, Sumo S, Bayrak ES et al (2013) Three-dimensional modeling of angiogenesis in porous biomaterial scaffolds. Biomaterials 34(12):2875-2887

3. Karageorgiou V, Kaplan DL (2005) Porosity of 3D biomaterial scaffolds and osteogenesis. Biomaterials 26(27):5474-5491

4. Hollister SJ (2005) Porous scaffold design for tissue engineering. Nat Mater 4(7):518-524

5. Hutmacher DW (2000) Scaffolds in tissue engineering bone and cartilage. Biomaterials 21(24):2529-2543

6. Ball JP, Mound BA, Monsalve A et al (2015) Biocompatibility evaluation of porous ceria foams for orthopedic tissue engineering. J Biomed Mater Res Part A 103(1):8-15

7. Tang F, Li L, Chen D et al (2012) Mesoporous silica nanoparticles: synthesis, biocompatibility and drug delivery. Adv Mater 24(12):1504-1534

8. Chia HN, Wu BM (2015) Recent advances in 3D printing of biomaterials. J Biol Eng 9(1):4

9. Chua CK, Leong KF, Cheah CM, Chua SW (2003) Development of a tissue engineering scaffold structure library for rapid prototyping. Part 1: investigation and classification. Int J Adv Manuf Technol 21:291-301

10. Lord EA, Mackay AL (2003) Periodic minimal surfaces of cubic symmetry. Curr Sci 85(3):346-362

11. Wohlgemuth M, Yufa N, Hoffman J, Thomas EL (2001) Triply periodic bicontinuous cubic microdomain morphologies by symmetries. Macromolecules 34(17):6083

12. Wang $Y(2007)$ Periodic surface modeling for computer aided nano design. Comput Aided Design 39(3):179

13. Kapfer SC, Hyde ST, Mecke K, Arns CH, Schröder-Turk GE (2011) Minimal surface scaffold designs for tissue engineering. Biomaterials 32:6875

14. Rajagopalan S, Robb RA (2006) Schwarz meets Schwann: design and fabrication of biomorphic tissue engineering scaffolds. Med Image Anal 2006(10):693

15. Zou X, Ren H, Zhu G et al (2013) Topology-directed design of porous organic frameworks and their advanced applications. Chem Commun 49(38):3925-3936

16. Zheng $X, F u Z$, Du K et al (2018) Minimal surface designs for porous materials: from microstructures to mechanical properties. J Mater Sci 53(4):10194-20208

17. Syam WP, Jianwei W, Zhao B, Maskery I, Elmadih W, Leach R (2018) Design and analysis of strut-based lattice structures for vibration isolation. Precis Eng 52:494-506

18. Von Schnering H, Nesper R (1991) Nodal surfaces of Fourier series: fundamental invariants of structured matter. Zeitschrift für Physik B Condensed Matter 83(3):407

19. http://www.msri.org/ (2018). Accessed on 1 Jan. 2018

20. https://www.geodict.com/ (2018). Accessed on 1 Jan. 2018
21. Abueidda DW, Al-Rub RKA, Dalaq AS, Lee DW, Khan KA, Jasiuk I (2016) Effective conductivities and elastic moduli of novel foams with triply periodic minimal surfaces. Mech Mater 95:102

22. Abueidda DW, Dalaq AS, Al-Rub RKA, Jasiuk I (2015) Micromechanical finite element predictions of a reduced coefficient of thermal expansion for 3D periodic architectured interpenetrating phase composites. Compos Struct 133:85

23. Lee DW, Khan KA, Al-Rub RKA (2017) Stiffness and yield strength of architectured foams based on the Schwarz primitive triply periodic minimal surface. Int J Plast 95:1

24. Dalaq AS, Abueidda DW, Al-Rub RKA, Jasiuk IM (2016) Finite element prediction of effective elastic properties of interpenetrating phase composites with architectured 3D sheet reinforcements. Int J Solids Struct 83:169

25. Eymard R, Gallouït T, Herbin Raphaèle et al (2002) Convergence of a finite volume scheme for nonlinear degenerate parabolic equations. Numer Math 92(1):41-82

26. Kabel $M$, Andrä H (2012) Fast numerical computation of precise bounds of effective elastic moduli. Berichte Fraunhofer ITWM 224:1-6

27. Rutka V, Wiegmann A (2006) Explicit jump immersed interface method for virtual material design of the effective elastic moduli of composite materials. Numer Algorithms 43(4):309

28. Yi Y, Zheng X, Fu Z, Wang C, Xu X, Tan X (2018) Multiscale modeling for predicting the stiffness and strength of hollow-structured metal foams with structural hierarchy. Materials 11(3):380

29. Hao M, Xiaoyang Z, Xuan L et al (2018) Simulation and analysis of mechanical properties of silica aerogels: from rationalization to prediction. Materials 11(2):214

30. Onck P, Andrews E, Gibson L (2001) Size effects in ductile cellular solids. Part I: modeling. Int J Mech Sci 43(3):681

31. Andrews E, Gioux G, Onck P, Gibson L (2001) Size effects in ductile cellular solids Part II: experimental results. Int J Mech Sci 43(3):701

32. Maskery I, Aremu AO, Parry L et al (2018) Effective design and simulation of surface-based lattice structures featuring volume fraction and cell type grading. Mater Design 155:220-232

33. Maskery I, Sturm L, Aremu AO et al (2018) Insights into the mechanical properties of several triply periodic minimal surface lattice structures made by polymer additive manufacturing. Polymer 152:62-71

34. A. International (2015) Standard test method for compressive properties of rigid plastics. ASTM International, Conshohocken

35. Giannitelli SM, Accoto D, Trombetta M et al (2014) Current trends in the design of scaffolds for computer-aided tissue engineering. Acta Biomater 10(2):580-594

36. Gibson LJ, Ashby MF (1999) Cellular solids: structure and properties. Cambridge University Press, Cambridge

37. Ashby M (2006) Philosophical transactions of the royal society of London a: mathematical. Phys Eng Sci 364(1838):15

38. Deshpande V, Ashby M, Fleck N (2001) Foam topology: bending versus stretching dominated architectures. Acta Mater 49(6):1035

Publisher's Note Springer Nature remains neutral with regard to jurisdictional claims in published maps and institutional affiliations. 